DART-HEP-94/01

March 1994

\title{
DYNAMICS OF WEAK FIRST ORDER PHASE TRANSITIONS*
}

\author{
Marcelo Gleiser \\ Department of Physics and Astronomy, Dartmouth College, Hanover, NH 03755
}

\begin{abstract}
The dynamics of weak vs. strong first order phase transitions is investigated numerically for $2+1$ dimensional scalar field models. It is argued that the change from a weak to a strong transition is itself a (second order) phase transition, with the order parameter being the equilibrium fractional population difference between the two phases at the critical temperature, and the control parameter being the coefficient of the cubic coupling in the free-energy density. The critical point is identified, and a power law controlling the relaxation dynamics at this point is obtained. Possible applications are briefly discussed.
\end{abstract}

PACS : 98.80.Cq, 64.60.Cn, 64.60.-i

* electronic mail: gleiser@peterpan.dartmouth.edu 
Of the many interesting possibilities raised by primordial phase transitions [1], the generation of the baryon number of the Universe during the electroweak transition has been extensively investigated following the seminal work of Kuzmin, Rubakov, and Shaposhnikov [2]. For the purpose of this paper, the important aspect of the electroweak phase transition is that it is, in most scenarios proposed so far, a first order phase transition. And, at least within the context of the standard model of particle physics, the transition is very possibly a weak one; the standard computation for nucleation of critical bubbles (see Refs. [3 - 5]) shows that the thin-wall approximation fails and that the bubbles are rather thick [6].

The possibility that the electroweak transition could be weakly first order has led Gleiser and Kolb (GK) to propose a novel mechanism by which such transitions evolve [7]. The transition would be characterized by a substantial phase mixing as the critical temperature $T_{c}$ (i.e. when the two phases are degenerate) is approached from above, followed by domain coarsening below $T_{c}$. (The slow cooling is provided by the expansion of the Universe.) GK modelled the dynamics of this phase mixing by estimating the fraction of the volume occupied by sub-critical (correlation volume) thermal fluctuations of each phase as a function of the temperature. They neglected the fact that these sub-critical fluctuations were unstable and thus found that the system would be equally populated by the two phases as it reached $T_{c}$. The results of Ref. [8 indicate that GK are at least qualitatively correct; there will be a regime in which the transition is weak enough that considerable phase mixing occurs even above $T_{c}$. (It is of course possible, although not directly relevant here, that this interesting regime lies beyond the validity of the perturbative evaluation of the electroweak effective potential. Presently this question does not appear to be resolved [9].)

Due to the complex nonequilibrium nature of the system, any analytical approach is bound to be severely limited. The need for a numerical investigation of this question 
is clear. This need is even more justified by noting that several of the gross features of the electroweak transition may appear in other unrelated physical systems such as nematic liquid crystals and certain magnetic materials. Moreover, numerical simulations of first-order transitions in the context of field theories (as opposed to discrete Ising models [10]) are scarse. Recent work has shown that the effective nucleation barrier is accurately predicted by homogeneous nucleation theory in the context of 2+1-dimensional classical field theory [11]. These results were obtained for strong transitions, in which the nucleation barrier $B$ was large. Nucleation was made possible due to the fairly high temperatures used in the simulations. (Recall that the decay time is proportional to $\exp (B / T) \cdot)$

In order to study how the weakness of the transition will affect its dynamics, the homogeneous part of the (coarse-grained Helmholtz) free-energy density is written as

$$
U(\phi, T)=\frac{a}{2}\left(T^{2}-T_{2}^{2}\right) \phi^{2}-\frac{\alpha}{3} T \phi^{3}+\frac{\lambda}{4} \phi^{4} .
$$

This free-energy density resembles the finite-temperature effective potential used in the description of the electroweak transition, where $\alpha$ is determined by the masses of the gauge bosons and $T_{2}$ is the spinodal instability temperature [2]. In the electroweak case the order parameter is the magnitude of the Higgs field and the effective potential is obtained after integrating out the gauge and fermionic degrees of freedom. Here, we will not be concerned with the limits of validity of the perturbative effective potential. The goal is to explore the possible dynamics of a transition with free-energy density given by Eq. 1, and use the results as suggestive of the behavior in the electroweak case. This freeenergy density is also similar to the de Gennes-Ginsburg-Landau free energy (with the elastic constants set to zero) used in the study of the isotropic-nematic transition in liquid crystals [12]. This transition is known to be weakly first-order; departures from the mean field prediction for the behavior of the correlation length were detected as the degeneracy temperature is approached from above, signalling the presence of "pre-transitional phe- 
nomena", due to long-wavelength fluctuations observed by light-scattering experiments 113.

It proves convenient to introduce dimensionless variables, $\tilde{x}=x \sqrt{a} T_{2}, \quad \tilde{t}=$ $t \sqrt{a} T_{2}, X=\phi / \sqrt{T_{2}}$, and $\theta=T / T_{2}$, so that we can write the Hamiltonian as

$$
\frac{H[X]}{\theta}=\frac{1}{\theta} \int d^{2} \tilde{x}\left[\frac{1}{2}|\tilde{\nabla} X|^{2}+\frac{1}{2}\left(\theta^{2}-1\right) X^{2}-\frac{\tilde{\alpha}}{3} X^{3}+\frac{\tilde{\lambda}}{4} X^{4}\right],
$$

where $\tilde{\alpha}=\alpha /\left(a \sqrt{T_{2}}\right)$, and $\tilde{\lambda}=\lambda /\left(a T_{2}\right)$. (From now on the tildes will be dropped unless a new quantity is introduced.) For temperatures above $\theta_{1}=\left(1-\alpha^{2} / 4 \lambda\right)^{-1 / 2}$ there is only one minimum at $X=0$. At $\theta=\theta_{1}$ an inflection point appears at $X_{\text {inf }}=\alpha \theta_{1} / 2 \lambda$. Below $\theta_{1}$ the inflection point separates into a maximum and a minimum given by $X_{ \pm}=$ $\frac{\alpha \theta}{2 \lambda}\left[1 \pm \sqrt{1-4 \lambda\left(1-1 / \theta^{2}\right) / \alpha^{2}}\right]$. At the critical temperature $\theta_{c}=\left(1-2 \alpha^{2} / 9 \lambda\right)^{-1 / 2}$ the two minima, at $X_{0}=0$ and $X_{+}$are degenerate. Below $\theta_{c}$ the minimum at $X_{+}$becomes the global minimum and the $X_{0}$-phase becomes metastable. Finally, at $\theta=1$ the barrier between the two phases disappears.

In order to study numerically the approach to equilibrium at a given temperature $\theta$, the coupling of the order parameter $X$ with the thermal bath will be modelled by a Markovian Langevin equation,

$$
\frac{\partial^{2} X}{\partial t^{2}}=\nabla^{2} X-\tilde{\eta} \frac{\partial X}{\partial t}-\frac{\partial U(X, \theta)}{\partial X}+\tilde{\xi}(x, t)
$$

where $\tilde{\eta}=\eta / \sqrt{a} T_{2}$ is the dimensionless viscosity coefficient, and $\tilde{\xi}=\xi / a T_{2}^{5 / 2}$ is the dimensionless stochastic noise with vanishing mean, related to $\eta$ by the fluctuationdissipation theorem, $\left\langle\xi(\vec{x}, t) \xi\left(\vec{x}^{\prime}, t^{\prime}\right)\right\rangle=2 \eta T \delta\left(t-t^{\prime}\right) \delta^{2}\left(\vec{x}-\vec{x}^{\prime}\right)$. The viscosity coefficient was set to unity in all simulations. The lattice spacing was also set to unity in all simulations. It turns out that in all cases of interest here the mean-field correlation length $\xi_{\text {cor }}^{-2}=U^{\prime \prime}\left(X_{0}, \theta\right)$ will be sufficiently larger than unity to justify this choice. In future work it would be interesting to see how to generalize the lattice renormalization conditions obtained in Ref. 11 for temperature independent potentials to the situation 
studied here. The Langevin equation was integrated using the fifth-degree Nordsiek-Geer algorithm, which allows for fast integration with high numerical accuracy [14]. The time step used was $\delta t=0.2$, and results were obtained with a square lattice with $L=64$. (Comparison with $L=40$ and $L=128$ produced negligible differences for our present purposes.) No dependence of the results was found on the time-step, random noise generator, and random noise seed.

The strategy adopted was to study the behavior of the system given by Eq. [2] at the critical temperature when the two minima are degenerate. The reason for this choice of temperature is simple. If at $\theta_{c}$ most of the system is found in the $X=0$ phase then as the temperature drops below $\theta_{c}$, one expects homogeneous nucleation to work; the system is well-localized in its metastable phase. This is what happens when a system is rapidly cooled below its critical temperature (rapid quench), so that it finds itself trapped in the metastable state. The large amplitude fluctuations which will eventually appear and grow are the nucleating bubbles. If, at $\theta_{c}$ one finds a large probability for the system to be in the $X_{+}$-phase, then considerable phase-mixing is occuring and homogeneous nucleation should not be accurate in describing the transition. Large amplitude fluctuations are present in the system before it is quenched to temperatures below $\theta_{c}$. For definiteness call the two phases the 0-phase and the +-phase. The phase distribution of the system can be measured if the idea of fractional area (volume in three dimensions) is introduced. As the field evolves according to Eq. 3, one counts how much of the total area of the lattice belongs to the 0 -phase with $X \leq X_{-}$(i.e. to the left of the maximum), and how much belongs to the + -phase with $X>X_{-}$(i.e. to the right of the maximum). Dividing by the total area one obtains the fractional area in each phase, so that $f_{0}(t)+f_{+}(t)=1$, indepedently of $L^{2}$.

The system is prepared initially in the 0 -phase, $f_{0}(0)=1$ and $f_{+}(0)=0$. Thus, the area-averaged value of the order parameter, $\langle X\rangle(t)=A^{-1} \int X d A$ is initially zero. 
The coupling with the thermal bath will induce fluctuations about $X=0$. By keeping $\lambda=0.1$ fixed, the dependence of $f_{0}(t), f_{+}(t)$, and $\langle X\rangle(t)$ on the value of $\alpha$ can be measured. Larger values of $\alpha$ imply stronger transitions. This is clear from the expression for $\theta_{c}$ which approaches unity as $\alpha \rightarrow 0$. (In the electroweak case, the same argument applies, as what is relevant is the ratio $\alpha^{2} / \lambda$; $\alpha$ is fixed but $\lambda$ increases as the Higgs mass increases.) The results are shown in Figure 1 for several values of $\alpha$ between $\alpha=0.3$ and $\alpha=0.4$. Each one of these curves is the result of averaging over 200 runs. The two important features here are the final equilibrium fraction in each phase and the equilibration time-scale. The approach to equilibrium can be fitted at all times to an exponential,

$$
f_{0}(t)=\left(1-f_{0}^{\mathrm{EQ}}\right) \exp \left[-\left(t / \tau_{\mathrm{EQ}}\right)^{\sigma}\right]+f_{0}^{\mathrm{EQ}}
$$

where $f_{0}^{\mathrm{EQ}}$ is the final equilibrium fraction and $\tau_{\mathrm{EQ}}$ is the equilibration time-scale. In Table 1 the values of $\tau_{\mathrm{EQ}}$ and $\sigma$ are listed for different values of $\alpha$. Note that the slot for $\alpha=0.36$ is empty. For this value of $\alpha$ the approach to equilibrium cannot be fitted at all times to an exponential; however, at large times it can be fitted to a power law,

$$
\left.f_{0}(t)\right|_{\alpha=0.36} \propto t^{-k},
$$

where $k$ is the critical exponent controlling the approach to equilibrium. A good fit is obtained for $k=0.25( \pm 0.01)$ as shown in Figure 2. Note that this is not the same as the dynamical critical exponent $z \simeq 2$, defined as $\tau_{\mathrm{EQ}}=\xi_{\text {cor }}^{z}$.

The fact that there is a critical slowing down of the dynamics for $\alpha \simeq 0.36$ is indicative of the presence of a second order phase transition near $\alpha \simeq 0.36$. Similar behavior has been found in liquid crystals in the neighborhood of the isotropic-nematic transition [15], and is typical of ferromagnetic materials near the Curie temperature. This transition reveals itself in a striking way if we define as an order parameter the equilibrium fractional difference $\Delta F_{\mathrm{EQ}}$, 


$$
\Delta F_{\mathrm{EQ}}=f_{0}^{\mathrm{EQ}}-f_{+}^{\mathrm{EQ}} .
$$

In Figure $3 \Delta F_{\mathrm{EQ}}$ is plotted as a function of $\alpha$. Clearly, there is a marked change in the behavior of the system around $\alpha=\alpha_{c} \simeq 0.36$. This curve is essentially identical to numerical results for the magnetization as a function of temperature in Ising models; the rounding is due to finite size effects. (See Fig. 2a in [10].) For $\alpha<\alpha_{c}$ the fractional area occupied by both phases in equilibrium is practically the same at 0.5 . There is considerable mixing of the two phases, with the system unable to distinguish between them. One may call this phase the symmetric phase with respect to the order parameter $\Delta F_{\mathrm{EQ}}$. For $\alpha>\alpha_{c}$ there is a clear distinction between the two phases, with the +phase being sharply suppressed. This may be called the broken-symmetric phase. As a consequence of this behavior a very clear distinction between a strong and weak transition is possible. A strong transition has $\alpha>\alpha_{c}$ so that the system is dominated by the 0 phase at $\theta_{c}$. For a weak transition neither phase clearly dominates and, as argued above, the dynamics should be quite different from the usual nucleation mechanism.

In order to understand the reason for the sharp change of behavior of the system near $\alpha_{c}$, in Figure 4 the equilibrium area-averaged order paramemeter $\langle X\rangle_{\mathrm{EQ}}$ and the inflection point $X_{\text {inf }}=\frac{\alpha \theta}{3 \lambda}\left[1-\sqrt{1-3 \lambda\left(1-1 / \theta^{2}\right) / \alpha^{2}}\right]$, are shown as a function of $\alpha$. Also shown is the rms amplitude of correlation-size fluctuations $X_{\mathrm{rms}}^{2}=\left\langle X^{2}\right\rangle_{T}-\langle X\rangle_{T}^{2}$, where $\langle\cdots\rangle_{T}$ is the normalized thermal average with probability distribution $P\left[X_{s c}\right]=\exp \left[-F\left[X_{s c}\right] / \theta\right]$. $F\left[X_{s c}\right]$ is the free energy of a gaussian-shape sub-critical fluctuation. For details see Ref. [16]. It is clear from this figure that the transition from weak to strong occurs as $\langle X\rangle_{\mathrm{EQ}}$ drops below $X_{\text {inf }}$. This result can be interpreted as an effective Ginzburg criterion for the weak-to-strong transition. It matches quite well the fact that the critical slowing down occurs for $\alpha \simeq 0.36$. This result is in qualitative agreement with the study of Langer et al. contrasting the onset of nucleation vs. spinodal decomposition for binary fluid and solid solutions [17], where it was found that the transition between the two regimes 
occurs roughly at the spinodal (i.e. at the inflection point). Even though $X_{\text {rms }}$ drops below $X_{\text {inf }}$ for a smaller value of $\alpha$, being a much less computer intensive quantitity to obtain, it should serve as a rough indicator of the weak-to-strong transition.

The present work raises many questions for future investigation. Apart from investigating the 3+1-dimensional case, and obtaining the critical exponent for the order parameter (as well as more accurate values for $\alpha_{c}, \tau_{\mathrm{EQ}}$ and $k$ ) using finite-size scaling techniques [10], $\Delta F_{\mathrm{EQ}} \propto\left(\alpha-\alpha_{c}\right)^{\beta}(\beta=1 / 8$ for the $d=2$ Ising model, and $\beta=1 / 2$ for mean field), it should be interesting to test if this behavior could be observed in the laboratory. A possible system would be an Ising magnetic film in the absence of an external field, heated to just above its measured Curie point. For the model studied here, this would correspond to $\alpha \lesssim \alpha_{c}$.

\section{ACKNOWLEDGMENTS}

I am grateful to W. E. Lawrence for many enlightening comments and to M. Alford, H. Müller, and Rudnei Ramos for useful discussions. I also thank F. F. Abraham for his guidance with the numerical work during the early stages of this investigation. This work is partially supported by a National Science Foundation grant No. PHYS-9204726. 


\section{REFERENCES}

1. E. W. Kolb and M. S. Turner, The Early Universe, Addison-Wesley (1990).

2. V. A. Kuzmin, V. A. Rubakov, and M. E. Shaposhnikov, Phys. Lett. 155B, 36 (1985). For a recent review see, A. Cohen, D. Kaplan, and A. Nelson, Ann. Rev. Nucl. Part. Sci. 43, 27 (1993).

3. For a review of homogeneous nucleation see, J. D. Gunton, M. San Miguel, and P. S. Sahni, in Phase Transitions and Critical Phenomena, edited by C. Domb and J. L. Lebowitz (Academic, London, 1983), Vol. 8.

4. S. Coleman, Phys. Rev. D15, 2929 (1977); C. Callan and S. Coleman, Phys. Rev. D16, 1762 (1977).

5. A. D. Linde, Nucl. Phys. B216, 421 (1983); [Erratum: B223, 544 (1983)]; for recent work, see e.g., L. P. Csernai and J. I. Kapusta, Phys. Rev. D46, 1379 (1992); M. Gleiser, G. Marques, and R. Ramos, Phys. Rev. D48, 1571 (1993); D. Brahm and C. Lee, Caltech preprint No. CALT-68-1881 (1993).

6. See, e.g., B. Liu, L. McLerran, and N. Turok, Phys. Rev. D46, 2668 (1992); M. Dine and S. Thomas, Santa Cruz preprint No. SCIPP 94/01.

7. M. Gleiser and E. W. Kolb, Phys. Rev. Lett. 69, 1304 (1992); Phys. Rev. D48, 1560 (1993).

8. M. Gleiser and G. Gelmini, in press, Nucl. Phys. B. Criticism to the GK approach can be found in this reference.

9. P. Arnold and L. Yaffe, Univ. of Washington preprint No. UW/PT-93-24, and refs. therein.

10. D. P. Landau in Finite Size Scaling and Numerical Simulations of Statistical Systems, edited by V. Privman (World Scientific, Singapore, 1990). 
11. M. Alford and M. Gleiser, Phys. Rev. D48, 2838 (1993); O. T. Valls and G. F. Mazenko, Phys. Rev. B42, 6614 (1990).

12. S. Chandrasekhar, Liquid Crystals, (Cambridge University Press, Cambridge [Second edition, 1992]).

13. T. W. Stinson and J. D. Litster, Phys. Rev. Lett. 25, 503 (1970); H. Zink and W. H. de Jeu, Mol. Cryst. Liq. Cryst. 124, 287 (1985).

14. A. Nordsiek, Math. Comp. 16, 22 (1962).

15. F. W. Deeg and M. D. Fayer, Chem. Phys. Lett. 167, 527 (1990); J. D. Litster and T. W. Stinson, J. App. Phys., 41, 996 (1970).

16. M. Gleiser and R. Ramos, Phys. Lett. B300, 271 (1993).

17. J. Langer, Physica 73, 61 (1974); J. Langer, M. Bar-on, and H. Miller, Phys. Rev. A11, 1417 (1975). Note that these authors studied transitions with a conserved order parameter, and thus with slower dynamics.

\section{List of Figures}

Figure 1. The approach to equilibrium for several values of $\alpha$.

Figure 2. Fitting $f_{0}\left(\theta_{c}\right)$ to a power law at large times for $\alpha=0.36$.

Figure 3. The fractional equilibrium population difference $\Delta F_{\mathrm{EQ}}$ as a function of $\alpha$.

Figure 4. Comparison between area-averaged field and location of the inflection point as a function of $\alpha$. Also shown are the location of the barrier, $X_{\mathrm{MAX}}$ and the rms fluctuation $X_{\text {rms }}$. 


\section{List of Tables}

Table 1. The values of the equilibration time-scales and the exponents for the exponential

fit of Eq. 4 for several values of $\alpha$. Also shown are the equilibrium fractions $f_{0}\left(\theta_{c}\right)$ and $f_{+}\left(\theta_{c}\right)$. Uncertainties are in the last digit. 
TABLE 1

\begin{tabular}{|c|c|c|c|c|}
\hline \hline$\alpha$ & $\tau_{\mathrm{EQ}}$ & $\sigma$ & $f_{0}\left(\theta_{c}\right)$ & $f_{+}\left(\theta_{c}\right)$ \\
\hline \hline 0.30 & 21.0 & 0.80 & 0.505 & 0.495 \\
0.33 & 40.0 & 0.80 & 0.514 & 0.486 \\
0.35 & 75.0 & 0.60 & 0.525 & 0.475 \\
0.36 & - & - & 0.580 & 0.420 \\
0.37 & 25.0 & 0.65 & 0.800 & 0.200 \\
0.38 & 15.0 & 0.80 & 0.870 & 0.130 \\
0.40 & 5.0 & 1.0 & 0.937 & 0.063 \\
\hline
\end{tabular}


This figure "fig1-1.png" is available in "png" format from: http://arxiv.org/ps/hep-ph/9403310v1 
This figure "fig1-2.png" is available in "png" format from: http://arxiv.org/ps/hep-ph/9403310v1 
This figure "fig1-3.png" is available in "png" format from: http://arxiv.org/ps/hep-ph/9403310v1 
This figure "fig1-4.png" is available in "png" format from: http://arxiv.org/ps/hep-ph/9403310v1 